\title{
CRISPR/Cas9-edited HSPC therapy for hemoglobinopathies
}

Humbert, O. et al. Sci. Transl. Med. 11, eaaw3768 (2019)

$\beta$-hemoglobinopathies are inherited disorders caused by mutations in the $\beta$-globin gene. Sickle cell disease, for example, is the result of deficient hemoglobin $(\mathrm{Hb})$; patients with $\beta$-thalassemia produce insufficient amounts of the oxygen-carrying protein. To date, allogeneic transplantation of hematopoietic stem and progenitor cells (HSPC) is the only curative treatment available for hemoglobinopathies, but alternative therapeutic approaches are under investigation. One promising option is to use gene-editing technologies to reactivate fetal $\mathrm{Hb}(\mathrm{HbF}) ; \mathrm{HbF}$ can then act as a substitute for aberrant adult $\mathrm{Hb}$. The findings of a new study describing persistent $\mathrm{HbF}$ reactivation after engraftment of CRISPR/Cas9-edited CD $34^{+}$HSPC in a nonhuman primate (NHP) autologous transplantation model might bring us a step closer to the clinical translation of gene-edited HSPC therapy to patients.

The gene-editing strategy was designed to generate mutations in the $\mathrm{HbF}$-associated locus (HBG) containing the binding site for B-cell lymphoma/leukemia 11A (BCL11A), an $\mathrm{HbF}$ repressor controlling the fetal to adult $\mathrm{Hb}$ switch. Three rhesus macaques were infused with CRISPR/Cas9-treated CD $34{ }^{+}$HSPCs after total body irradiation. The number of gene-edited nucleated cells stabilized after 1 month (8-27\% of cells in peripheral blood $(\mathrm{PB})$ during the entire follow-up period of $>$ 1year), resulting in persistent $\mathrm{HbF}$ reactivation in transplanted animals. Compared with control transplant recipients showing $<1.5 \%$ of cells expressing $\mathrm{HbF}$ ( $\mathrm{F}$ cells) in $\mathrm{PB}$ at 250 days after treatment, all CRISPR/Cas9-edited animals expressed $6-18 \%$ of $\mathrm{F}$ cells during follow-up. HbF reactivation was further confirmed by longitudinal measurement

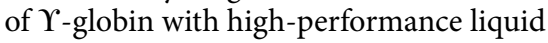
chromatography (HPLC).

Similar results were observed in a second cohort of three rhesus macaques infused with a CRISPR/Cas9-edited stem cell-enriched CD34+ ${ }^{+}$subpopulation of $\mathrm{CD} 34^{+} \mathrm{CD} 90^{+} \mathrm{CD} 45 \mathrm{RA}^{-}$cells, although the number of gene-edited cells transplanted in the second cohort was reduced by
10 fold. These results confirm that the $\mathrm{CD} 34^{+} \mathrm{CD} 90^{+} \mathrm{CD} 45 \mathrm{RA}^{-}$subset is the main cell type contributing to multilineage longterm engraftment. Targeting that subset could be a new strategy to reduce the need for editing reagents without affecting engraftment, hematopoietic reconstitution, or $\mathrm{HbF}$ reactivation.

So far, the transplanted rhesus animals have been monitored for up to 1.5 years with no adverse effects detected. In the discussion of the study, the investigators conclude: "The conservation of the CD $34{ }^{+} \mathrm{CD} 90^{+} \mathrm{CD} 45 \mathrm{RA}^{-}$phenotype and the $H B G$ CRISPR-Cas9 gRNA target site between NHP and human, combined with the use of a highly clinically relevant largeanimal model for stem cell gene therapy and transplantation, should facilitate the direct translation of this approach to patients."

Alexandra Le Bras

Published online: 9 September 2019 https://doi.org/10.1038/s41684-019-0406-7

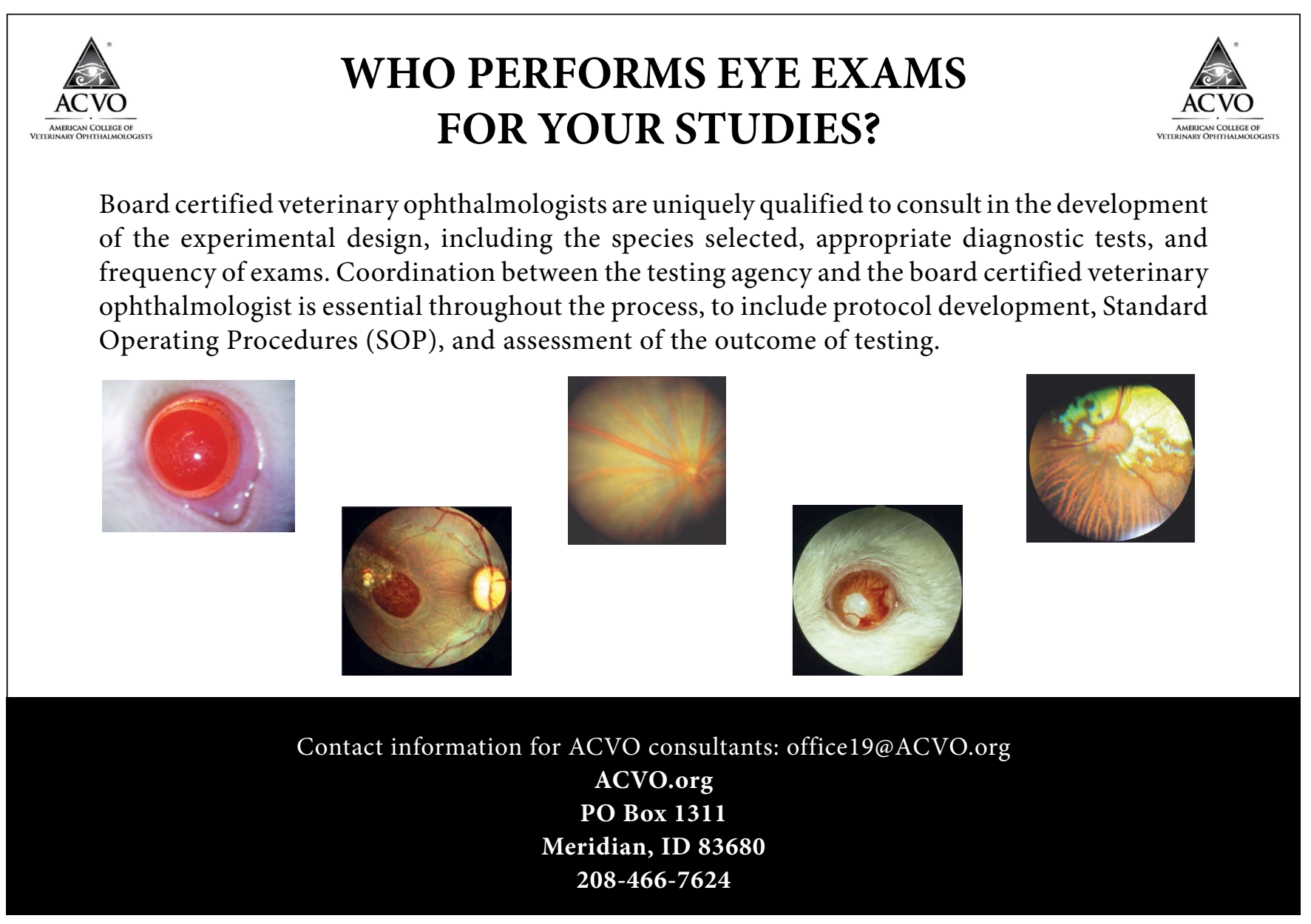

